\title{
An Efficient Node Localization Approach with RSSI for Randomly Deployed Wireless Sensor Networks
}

\author{
Xihai Zhang, Junlong Fang, and Fanfeng Meng \\ School of Electrical and Information Engineering, Northeast Agricultural University, Harbin 150030, China \\ Correspondence should be addressed to Junlong Fang; jlfang@neau.edu.cn
}

Received 12 December 2015; Revised 20 February 2016; Accepted 6 March 2016

Academic Editor: Iickho Song

Copyright ( 2016 Xihai Zhang et al. This is an open access article distributed under the Creative Commons Attribution License, which permits unrestricted use, distribution, and reproduction in any medium, provided the original work is properly cited.

\begin{abstract}
An efficient path planning approach in mobile beacon localization for the randomly deployed wireless sensor nodes is proposed in this paper. Firstly, in order to improve localization accuracy, the weighting function based on the distance between nodes is constructed. Moreover, an iterative multilateration algorithm is also presented to avoid decreasing the localization accuracy. Furthermore, a path planning algorithm based on grid scan which can traverse entirely in sensor field is described. At the same time, the start conditions of localization algorithm are also proposed to improve localization accuracy. To evaluate the proposed path planning algorithm, the localization results of beacon nodes randomly deployed in sensor field are also provided. The proposed approach can provide the deployment uniformly of virtual beacon nodes among the sensor fields and the lower computational complexity of path planning compared with method which utilizes only mobile beacons on the basis of a random movement. The performance evaluation shows that the proposed approach can reduce the beacon movement distance and the number of virtual mobile beacon nodes by comparison with other methods.
\end{abstract}

\section{Introduction}

Wireless sensor networks (WSNs) are closely associated with the physical phenomena in their surroundings. The gathered information needs to be associated with the position of sensor nodes to provide an accurate view of the observed sensor field. The localization is important in most applications, such as environment sensing, search and rescue, and geographical routing and tracking; the position of each node should be known [1]. These requirements motivate the development of efficient localization algorithms for WSNs.

Over the course of the past decade, there have been a large number of researches on localization for WSNs [2-8]. They share the same main idea that nodes with unknown coordinates are utilized by one or more GPS-equipped nodes with known coordinates in order to estimate their positions. Most of these works consider the static beacon. While GPS provides highly accurate location information, it may not be feasible for most randomly deployed WSNs. Firstly, GPS available for WSNs are very costly, exceeding the cost of a sensor node. Moreover, GPS operation has a high energy consumption profile, which may impose additional constraints on the lifetime of WSNs. Furthermore, WSNs are usually static and localization algorithms may be required to run only during initialization of the network. Consequently, GPS operation may not be cost-effective for many WSNs realizations.

Therefore, to obtain location information, we need a technique which incurs lesser cost and provides more accurate location. A promising method to localize randomly deployed WSNs is to use one mobile beacon [9-16]. The localization approach using mobile beacon utilizes a beacon node equipped with GPS to traverse the region of interest (ROI). This beacon node broadcasts periodically the packets including its position, and unknown nodes estimate their positions using the received packets. The problem of localization by mobile beacon in WSNs has attracted extensive interest in the literatures [17-24]. Sichitiu and Ramadurai [12] propose a range-based localization method in which the sensor nodes estimate their positions by applying an RSSI technique. Xia and Chen [19] propose a TDOA-based localization scheme with mobile anchors in which the sensor nodes perform trilateration to estimate their positions. Galstyan et al. [11] propose a range-free mobile anchor-based localization scheme based on radio connectivity constraints 
to reduce the uncertainty of the estimated sensor location. Dong and Severance [23] develop an iterative localization approach based on the mobile anchor scheme in which the localization accuracy is progressively improved each time a new beacon message is obtained from an anchor node. Kim and Lee [24] propose a novel range-based localization scheme which involves a movement strategy with a low computational complexity of mobile beacon, called mobile beaconassisted localization (MBAL). MBAL also applies RSSI for ranging to get the distance between nodes or between each node and the mobile beacon to assist localization of all nodes.

However, all approaches of mobile-assisted localization face the same problem, which is the optimum beacon path selection problem. Notice that the problem is quite difficult since the position of unknown nodes is not known. For example, some approaches [12] do not propose any specific movement strategy for the path of mobile beacon, and some approaches [25] just suggest that the beacon moves in the straight lines and then a loop. The random way point and Monte Carlo localization mobile model are utilized in most of localization algorithms based on a mobile beacon [26]. However, for those mobile models, the uniform deployment of virtual beacon in the sensor field is hard to realize, which results in emergence of localization blind area and then reduces localization accuracy. Although much effort is being spent on improving these weaknesses, the effective method has yet to be developed.

Therefore, a path planning algorithm based on grid scan which is to traverse entirely in sensor field is proposed in this paper. In order to make the beacon nodes closer to the unknown node have greater weight and to improve the localization accuracy, the weighting function is constructed based on the distance between the nodes. Furthermore, an iterative multilateration algorithm is also proposed to avoid decrease in the localization accuracy when the unknown node position is estimated. At the same time, the start conditions of localization algorithm are also proposed to improve localization accuracy. To evaluate the path planning algorithm based grid scan, the results of static beacon randomly deployed and RWP mobile path in sensor field are also provided in this paper.

\section{Localization in Sensor Network}

2.1. RSSI. Generally, the localization algorithms have been proposed, which can be mainly classified into two categories: range-based and range-free. Several range-based techniques estimate an unknown node distance by three or more beacon nodes. Based on the range information, the location of a node is determined. Some of the range-based localization algorithms include received signal strength indicator (RSSI), angle of arrival (AOA), time of arrival (TOA), and time difference of arrival (TDOA) [27]. On the other hand, range-free algorithms, such as Centroid Localization Algorithm, Distance Vector-Hop (DV-Hop), Approximate Pointin-Triangulation Test (APIT), and Rendered Path, only use the connectivity or proximity information to localize the unknown nodes. The most common range-based technique

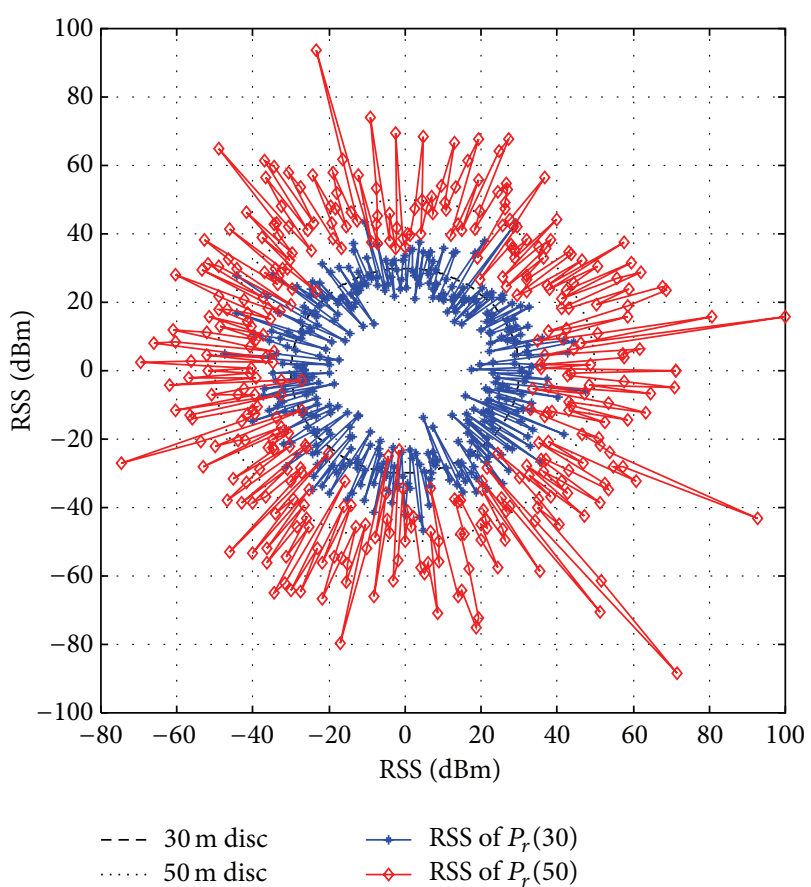

FIgURE 1: Relationship between RSS and distance.

is based on RSSI measurements. Since each sensor node is equipped with a radio and in most cases is able to send the received signal strength of an incoming packet, the main idea is to estimate the distance of a transmitter to a receiver using the power of received signal, knowledge of the transmitted power, and the path loss model.

In this scheme, the beacon node broadcasts periodically the messages including its own position, which are used to estimate the distance from the beacon node to unknown node. The power of the received signal is communicated by the transceiver circuitry through the RSSI. The received signal strength from sensor node $i$ at node $j$ at time $t$ is represented by $P_{r}(d)$, which is formulated as

$$
P_{r}(d)=P_{T}-\operatorname{PL}\left(d_{0}\right)-10 \eta \log _{10}\left(\frac{d}{d_{0}}\right)+X_{\delta},
$$

where $P_{r}(d)$ is the received signal power, $P_{T}$ is the transmit power, $\operatorname{PL}\left(d_{0}\right)$ is the path loss for reference distance of $d_{0}, \eta$ is the attenuation constant, and $X_{\delta}=N\left(0, \delta^{2}\right)$ is the uncertainty factor due to multipath and shadowing. Generally, the typical value of parameters is as follows: $P_{T}=0 \sim 4 \mathrm{dBm}$, $\operatorname{PL}\left(d_{0}\right)=55 \mathrm{~dB}\left(d_{0}=1 \mathrm{~m}\right), \eta=(2,4)$, and $\delta=(4,10)$.

The accuracy of the RSSI-based ranging technique is limited. Firstly, the effects of shadowing and multipath as modeled by the term $X_{\delta}$ in (1) may be severe and require multiple ranging measurements. For example, the parameters of (1) are as follows: $P_{T}=0, \operatorname{PL}\left(d_{0}\right)=55 \mathrm{~dB}, d_{0}=1 \mathrm{~m}, \eta=4$, $\delta=4$, and $d=30 \mathrm{~m}, 50 \mathrm{~m}$, which is applied in Section 4; then the relationship between the RSS and the node distance is illustrated in Figure 1. It shows that the receiving node which is far away from the sending node has relatively large impact on $X_{\delta}$. Therefore, the estimated distance based on RSSI has 


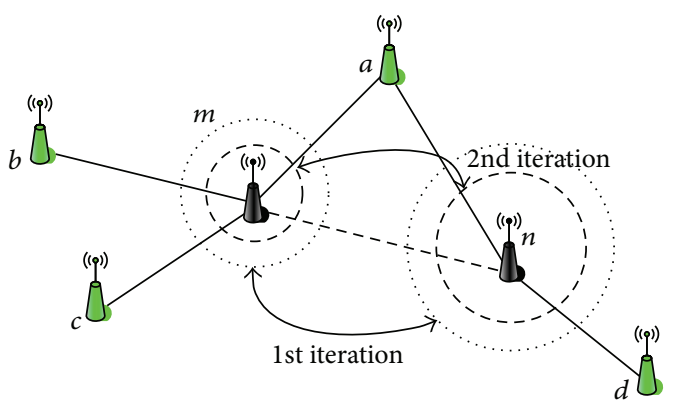

FIGURE 2: Iterative multilateration.

features such as the localization accuracy being high for the relatively near distance of nodes and the localization error being large for the relatively far distance of nodes.

Hence, in order to make the beacon nodes closer to the unknown nodes have greater weight and improve the localization accuracy of unknown node, the weighting function $\left(w(n)=1 / d_{i j}^{2}\right.$, with $d_{i j}$ being the distance from node $i$ to node $j$ ) is constructed based on the distance between the nodes in this paper.

2.2. Iterative Multilateration Algorithm. Range-based localization techniques exploit multiple pairwise range measurements to estimate the locations of unknown nodes. Generally, three mathematical techniques are used for calculating the position of a receiver from signals received from several transmitters: triangulation, trilateration, and maximum likelihood multilateration. The triangulation allows the unknown node to calculate its position by measuring two directions towards two beacon nodes. Since the positions of beacon nodes are known, it is therefore possible to construct a triangle where one of the sides and two of the angles are known, with the unknown node at the third point. This information is enough to define the triangle completely and hence deduces the position of unknown node. The trilateration requires the distance between the receiver and transmitter to be measured. This can be done using a received signal strength indicator (RSSI), ToA, and so forth. The position of unknown node is estimated by three pairwise distances from the unknown node to different beacon nodes. Multiple range measurements between a node and its different neighbors can be used to improve the localization accuracy. The trilateration technique fails to provide an accurate estimate of position if the distance measurements are noisy. Instead, maximum likelihood estimation method is necessary to incorporate distance measurements from multiple neighbor nodes. However, usually, WSNs are deployed with a limited number of beacon nodes. Furthermore, because of ranging errors, more than three beacon nodes are required for accurate location estimation. Hence, the unknown node location can be estimated through the location estimates of neighbor nodes. Therefore, a multihop network of sensors can be localized with the help of a subset of beacon nodes. This procedure is called iterative multilateration.

Iterative multilateration is illustrated in Figure 2, where two unknown nodes $m$ and $n$ are surrounded by four beacon nodes, $a, b, c$, and $d$. Note that node $m$ is surrounded by three beacon nodes ( $a, b$, and $c)$, whereas node $n$ has only two beacon nodes ( $a$ and $d$ ) as neighbors. Since node $m$ can communicate with at least three beacon nodes, the multilateration localization can be used to estimate its position. However, multilateration cannot be applied to node $n$, which requires additional distance information to a node with known location. In this case, iterative multilateration is used, where node $m$ first estimates its position and becomes a beacon node. Using the additional information from node $m$, node $n$ can perform multilateration and calculate its position. This information can in turn be utilized by node $m$ to further improve the accuracy of its estimation. As a result, the uncertainty in location estimation as shown by the circles around nodes $m$ and $n$ can be decreased at each iteration. Hence, for improving localization accuracy, iterative multilateration algorithm is used in this paper.

\section{Path Planning Algorithm Based on Grid Scan}

The range-based localization algorithms are discussed so far by utilizing ranging measurements from nodes with fixed locations. Consequently, the localization accuracy is inherently limited depending on the network topology and the deployment strategy. Since a uniform deployment of beacon nodes is not feasible in practice, some portions of the network may have a lower density of beacon and the neighbors of a node may not be sufficient.

For improving the localization accuracy and reducing the cost of network construction, a localization technique based on a mobile beacon is proposed in $[24,28]$, but the mobile beacon path planning is not referred to. Next, a path planning algorithm based on grid scan which is the entire traverse in sensor field is proposed in this paper.

The sensor field is divided by the grid area which is shown in Figure 3. And the area of a grid cell $s_{g}=l_{g}^{2}$, where $l_{g}$ denotes the edge length of a grid cell. The virtual beacon nodes are deployed when the mobile beacon node is moving at each vertex of cell grid. Hence, the mobile beacon path planning based on grid scan meets the requirements of complete coverage of sensor field. When the maximum communication radius of mobile beacon is defined, the quantity demand of virtual beacon nodes around the unknown node can be met by adjusting the edge length of a grid cell.

For the path planning based on grid scan, the mobile beacon is required through all vertexes of the grid cell. Here, the entire traverse path of the mobile beacon is denoted by the undirected graph $\mathrm{TG}=(V, E)$,

$$
\begin{aligned}
& V=\left\{v_{i}\right\}, \quad v_{i}=\left\{v_{i j}\right\} ; \\
& E=\left\{e^{i j}\right\}, \quad e^{i j}=\left\{e_{u v}^{i j}\right\},
\end{aligned}
$$

where $i=1, \ldots, m, j=1, \ldots, n, u=1, \ldots, m, v=1, \ldots, n, V$ denotes the traversing of the vertex set and the edge set, and $E$ denotes the distance set of the current vertex to other vertexes. The entire traverse path depended on the deploy cycle of the virtual beacon nodes and the localization speed. 


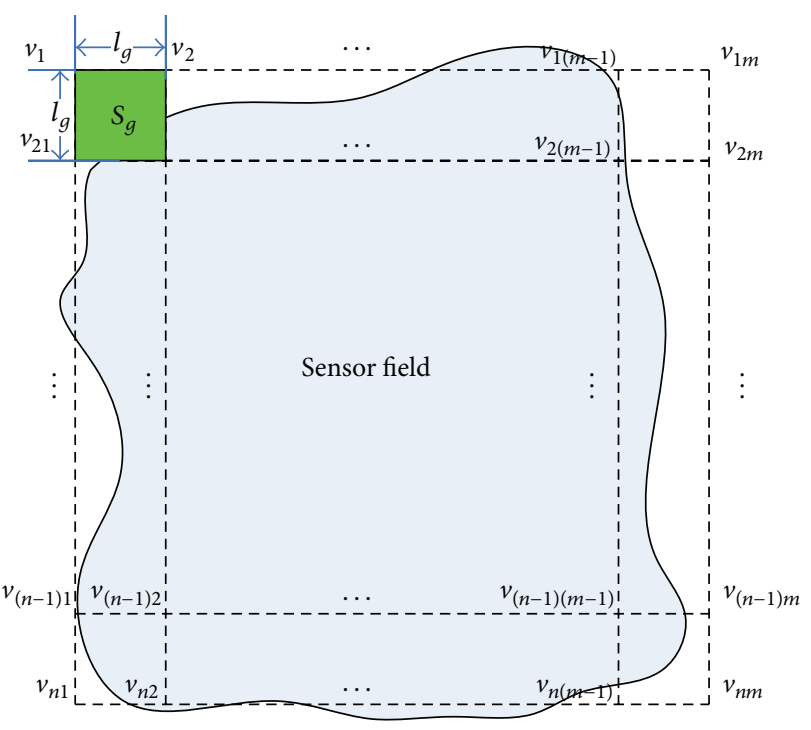

FIgURE 3: Grid cells of sensor field.

In this paper, $P_{\min }(V)$ denotes the shortest entire traverse path of the mobile beacon node; then

$$
\operatorname{dis}\left(P_{\min }(V)\right)=\sum_{e \in E} w(e)
$$

where $w(e)$ denotes the distance between the two grid vertexes where the mobile beacon node has been passed.

Theorem 1. For the mobile beacon path planning based on grid scan, with the shortest traverse path length of the mobile beacon node the following holds:

$$
\operatorname{dis}\left(P_{\min }(V)\right)=(m \cdot n-1) l_{g} .
$$

Proof. if $P(V)$ denotes one of some entire traverse paths, then this path can be taken as one path tree, and the following holds:

$$
\xi(P(V))=v(P(V))-1,
$$

where $\xi(P(V))$ is the number of edges, $v(P(V))$ is the number of vertexes, and $v(P(V))=m \cdot n$. Then $\operatorname{dis}(P(V))$ can be denoted as

$$
\operatorname{dis}(P(V))=\sum_{k=1}^{\xi(P(V))} w\left(e_{k}\right) \geq \xi(P(V))\left(w_{\min }\left(e_{k}\right)\right),
$$

where $e_{k} \in E$ and $w_{\min }\left(e_{k}\right)$ denotes the minimum value of $e_{k}\left(w_{\min }\left(e_{k}\right)=l_{g}\right)$. By substituting (5) and $w_{\min }\left(e_{k}\right)$ into (6), we can obtain

$$
\operatorname{dis}(P(V))=(m \cdot n-1) l_{g} ;
$$

then $\operatorname{dis}\left(P_{\min }(V)\right)=(m \cdot n-1) l_{g}$; the proof is completed.

There are many ways to obtain entire traverse path to hold in formula (4). However, a shortest entire traverse path based on grid scan can reduce the complexity of mobile beacon node's implementation. For the entire traverse path of the mobile beacon in sensor field, this algorithm has adaptability to irregular sensor field and the better effectiveness of location. At the same time, the realization of algorithm is easy by adjusting fewer parameters. Therefore, a shortest entire traverse path based on grid scan is proposed in this paper. However, in the process of positioning, due to the longer deployment period of virtual beacon node, the localization time is longer than that of the static beacon node localization algorithm. Because the unknown nodes are likely to not receive timely all the reference localization information from some neighbor beacons, the unknown nodes begin to estimate positions for themselves, which can cause some loss of localization accuracy. To receive the more reference localization information from the neighbor beacon nodes and improve the localization accuracy, we set the following two conditions of localization start.

\section{Condition 1. Consider}

$$
n_{\text {vitual beacon }} \geq k \cdot n_{\text {vitual beacon_threshold }} \quad(k>1) .
$$

Condition 2. Consider

$$
\begin{aligned}
n_{\text {all }} & \geq n_{\text {threshold }} \\
n_{\text {vitual beacon }} & \geq n_{\text {vitual beacon_threshold }},
\end{aligned}
$$

where $n_{\text {vitual beacon }}$ is the number of virtual beacon nodes to be received by unknown nodes, $n_{\text {vitual beacon_threshold }}$ is the threshold of virtual beacon to be set, $n_{\text {all }}=n_{\text {vitual beacon }}+$ $n_{\text {unknown node }}$ is the total number of reference nodes, $n_{\text {threshold }}$ is the threshold of reference nodes to be set, and $n_{\text {unknown node }}$ is the total numbers of unknown nodes which have been positioned and received by the nodes to be positioned.

If the total number of neighbor beacon nodes is more than

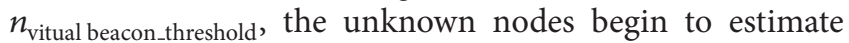
their location according to Condition 1. Then after the location of those nodes is obtained, they begin to send the packets including their locations. After the unknown nodes which do not meet the conditions of localization having received previous packets including location, they begin to estimate their location according to Condition 2.

\section{Simulation Experiments}

In this section, simulation experiments are conducted to study the accuracy of path planning algorithm based on grid scan in Matlab. For this comparison, we illustrate the results of two localization approaches in terms of the average number of neighbor beacon nodes, the number of unresolved localization nodes, and localization error, that is, the approach of static beacon randomly deployed and the approach of RWP mobile model. Unless otherwise noted, a rectangular $100 \mathrm{~m}$ $\times 100 \mathrm{~m}$ sensor field is selected, where nodes are deployed randomly in the simulation experiments.

4.1. Localization Experiments by Beacons Randomly Deployed. In the following, we present the impact of beacon node's number on the positioning performance. The neighbor relationship of nodes is shown in Figures 4(a), 4(c), and 4(e) and 


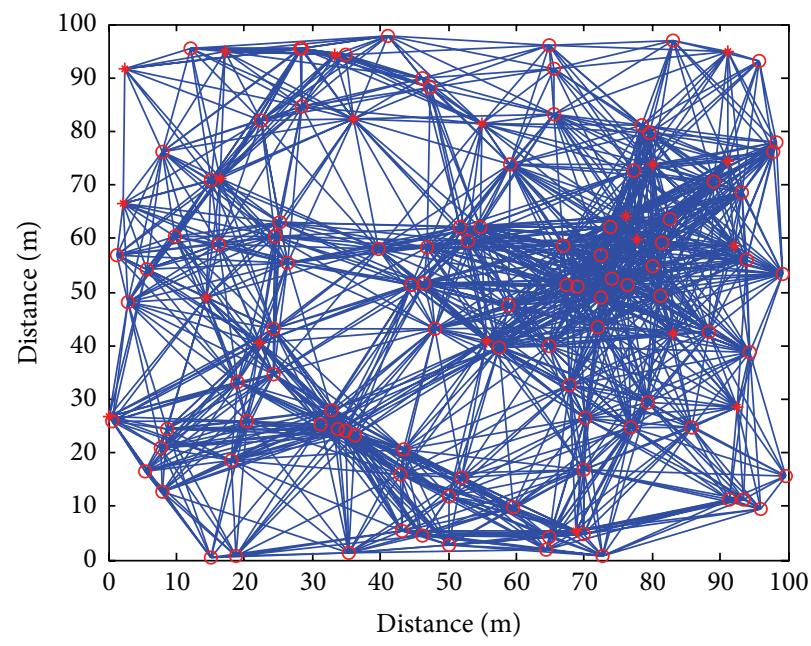

(a)

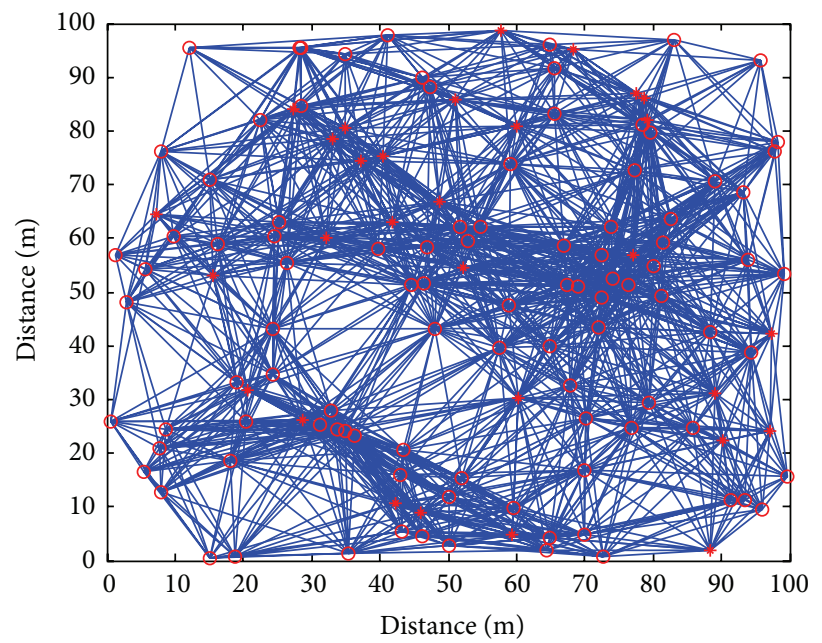

(c)

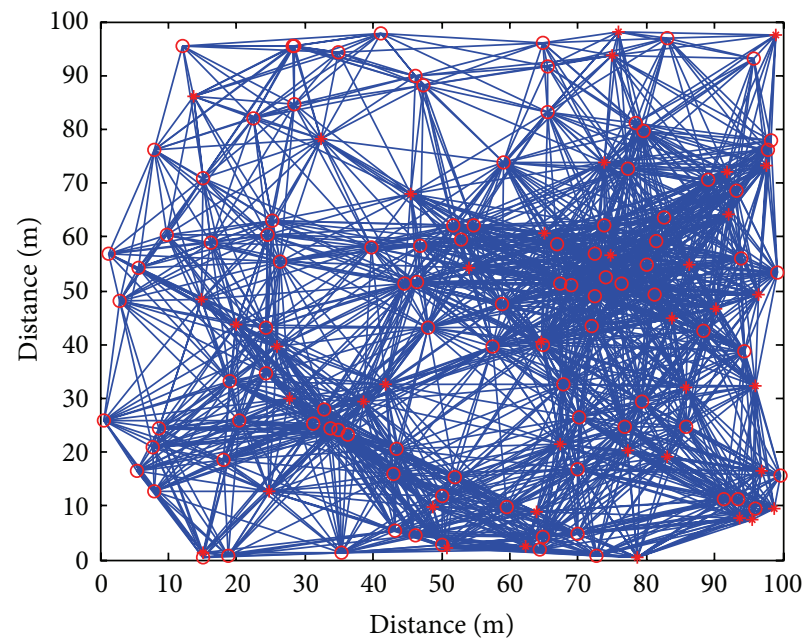

(e)

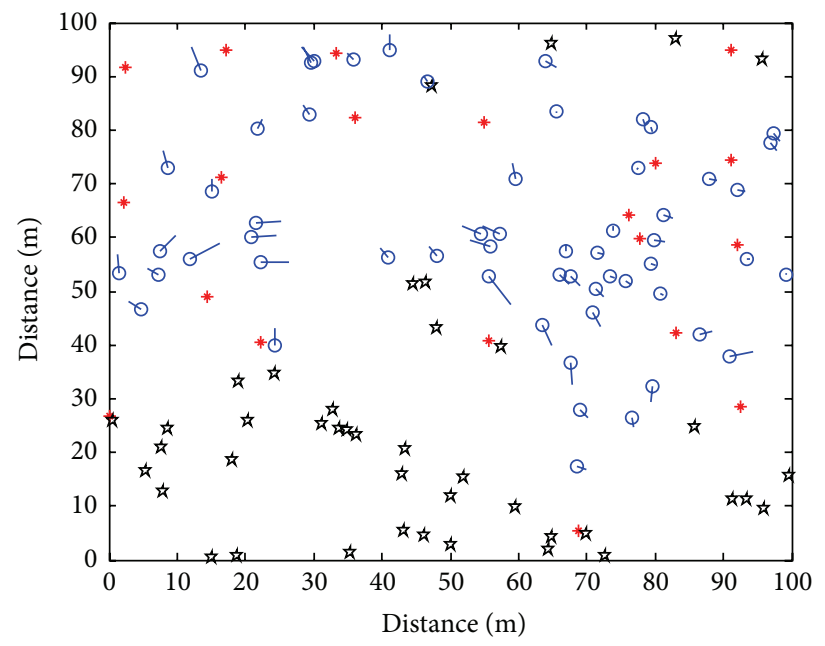

(b)

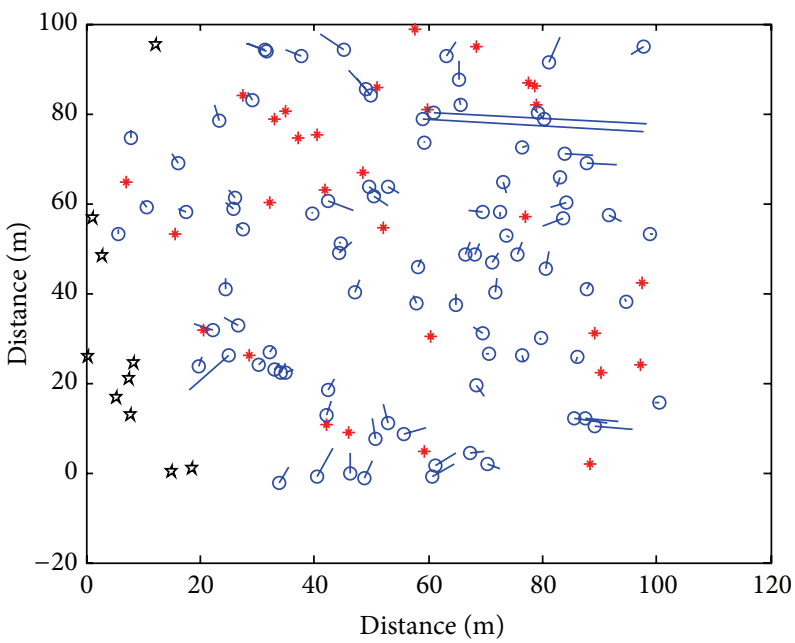

(d)

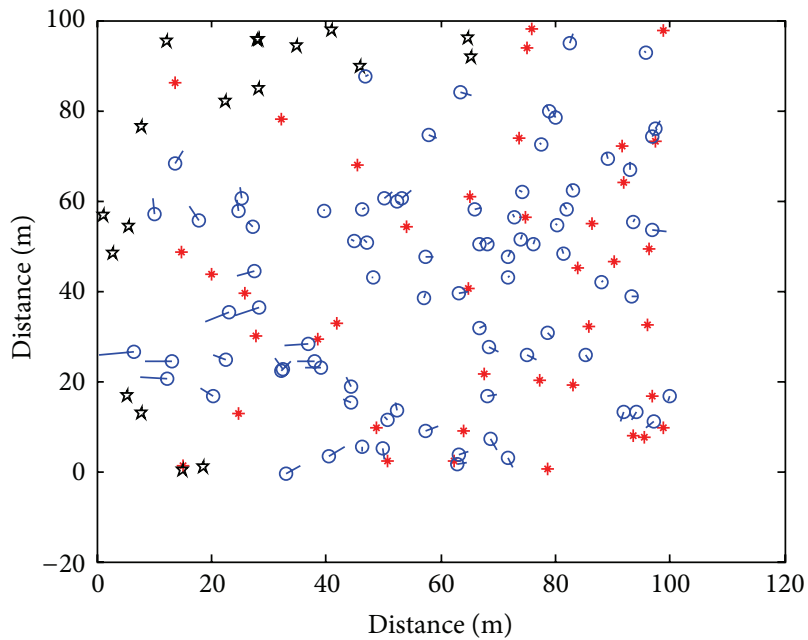

(f)

FIgURE 4: Connectivity and localization effect ((a), (c), and (e) are the neighbor relationship of nodes for the beacon node's number of 20, 30 , and 40, resp. (b), (d), and (f) are the localization error for the beacon node's number of 20, 30, and 40, resp. The communication radius is always $30 \mathrm{~m})$. 
TABLE 1: Contrast of two kinds of localization scheme.

\begin{tabular}{|c|c|c|c|c|}
\hline $\begin{array}{l}\text { Deployment pattern of beacon } \\
\text { node }\end{array}$ & $\begin{array}{l}\text { Average connectivity } \\
\text { of network }\end{array}$ & $\begin{array}{c}\text { Average number of } \\
\text { neighbor beacon } \\
\text { nodes }\end{array}$ & $\begin{array}{l}\text { Nodes of unresolved } \\
\text { localization }\end{array}$ & Localization error \\
\hline $\begin{array}{l}\text { Case } 1 \text { : beacon randomly } \\
\text { deployed (communication radius } \\
=30 \mathrm{~m} \text {; beacon nodes }=25)\end{array}$ & 25.008 & 4.216 & 28 & 0.12441 \\
\hline $\begin{array}{l}\text { Case 2: mobile beacon node } \\
\text { (communication radius }=30 \mathrm{~m} \text {; } \\
\text { number of beacon nodes }=25 \text { ) }\end{array}$ & 26.208 & 4.992 & 1 & 0.08767 \\
\hline
\end{tabular}

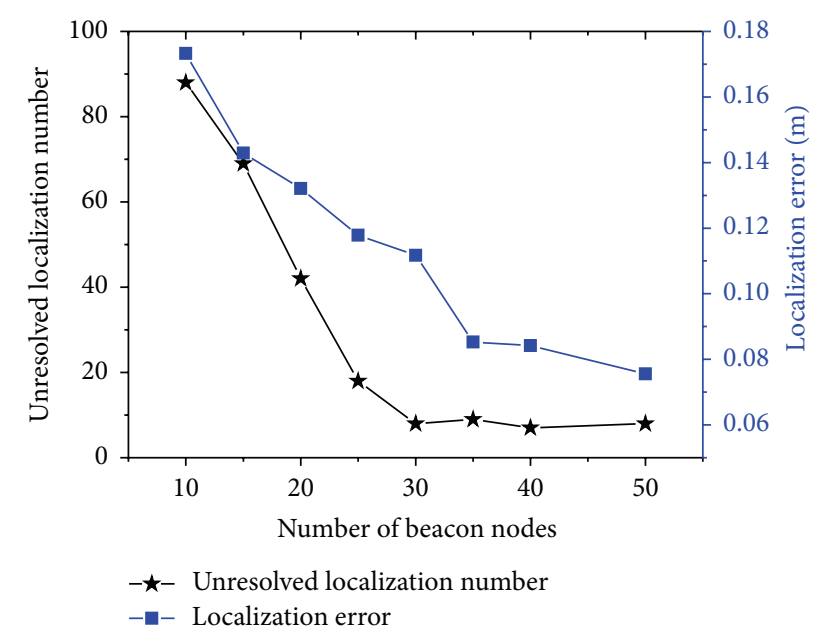

FIGURE 5: Relationship between localization effect and number of beacon nodes.

the localization error in Figures 4(b), 4(d), and 4(f) for the beacon node's number of 20,30 , and 40 , respectively. The node's communication radius is set to $30 \mathrm{~m}$. In this paper, unless otherwise noted, the dot "O" represents the actual position of unknown node, the asterisk " $*$ " represents the position of beacon node, and the pentagram " 23 " represents the unresolved localization node. Those figures clearly show that the beacon node's number affects significantly the connectivity and localization effect.

The relationship of the localization error and the unresolved localization number at different numbers of beacon is shown in Figure 5. As shown in Figure 5, at 10 beacon nodes, the 88 unknown nodes are not located successfully and the localization error is also above 0.17 . Increasing the beacon nodes number results in a rapid decrease in localization error and the unresolved localization number. However, when the beacon nodes exceed 30, the unresolved localization number tends to be stabilized. Even though the beacon nodes reach $50,10 \%$ nodes are not located successfully still. The reason is that the beacon nodes are not deployed uniformly among the sensor fields. Hence, in those simulation conditions, to illustrate the advantages of mobile beacons, we can set 25 virtual beacon nodes in the proposed mobile path.

We present the impact of beacon node's number on the positioning performance.
Second, we present the impact of communication radius on the positioning performance. The localization error and the neighbor relationship of nodes for different communication radius are shown in Figure 6. The neighbor relationship of nodes is shown in Figures 6(a), 6(c), and 6(e) and the localization error in Figures 6(b), 6(d), and 6(f), for the communication radius of 20,30 , and 40 , respectively. The number of beacon nodes is set to $30 \mathrm{~m}$. It is observed that the communication radius is heavily affected by the connectivity and localization effect.

The relationship of the localization error and the number of unresolved localization nodes at different communication radius is shown in Figure 7. As shown in Figure 7, at $10 \mathrm{~m}$ communication radius, all the unknown nodes are not located successfully. Increasing the communication radius results in a rapid decrease in localization error and the unresolved localization number. However, when the communication radius exceeds $40 \mathrm{~m}$, all the unknown nodes are located successfully and the localization error tends to be stabilized. Therefore, in these simulation conditions, the communication radius is set $30 \mathrm{~m}$ in the proposed mobile path in order to reduce power consumption of node.

4.2. Localization Experiments by a Mobile Beacon. In this section, according to preliminary experiments by beacons randomly deployed, the advantage of path planning algorithm based on a mobile beacon is illustrated.

Firstly, to illustrate the localization performance of proposed scheme by a mobile beacon, the localization method by beacon deployment randomly is also presented in simulation. In both cases, we all set 25 virtual beacon nodes and $30 \mathrm{~m}$ communication radius with the same unknown nodes in the same size sensor field. According to Section 3, the scan line divides the square deployment area into $n$ by $n$ subsquares ( $n=4, l_{g}=5 \mathrm{~m}$ in our case) and connects their vertexes using straight lines. Therefore, the 25 virtual beacon nodes are deployed uniformly in the sensor field. The node deployment is shown in Figures 8(a) and 8(b), the neighbor relationship of nodes is shown in Figures 8(c) and 8(d), and the localization error is shown in Figures 8(e) and 8(f).

The results are summarized in Table 1. In both cases, the average connectivity of network is little different. The average neighbor beacon number (Case 2) is obviously better than localization scheme by beacon deployment randomly. The nodes number of unresolved localization is only one 


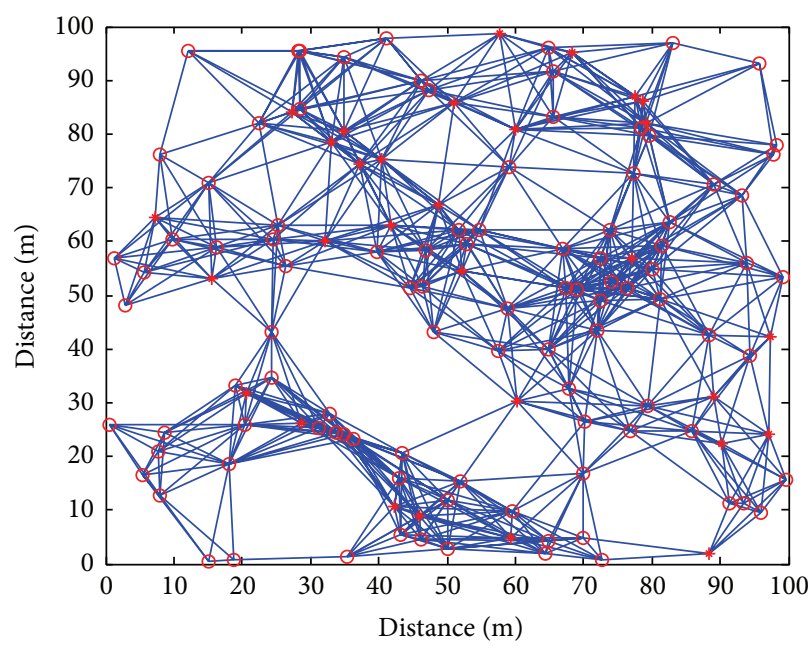

(a)

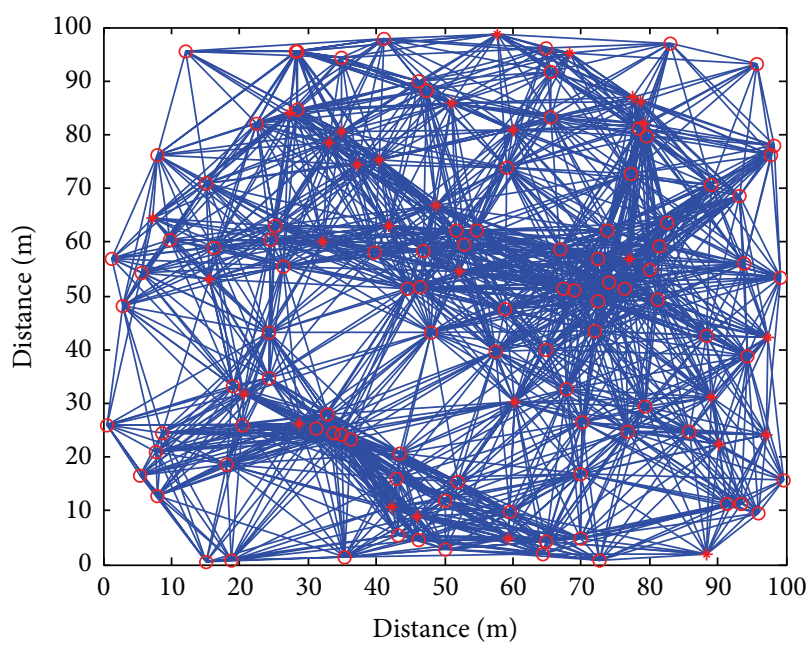

(c)

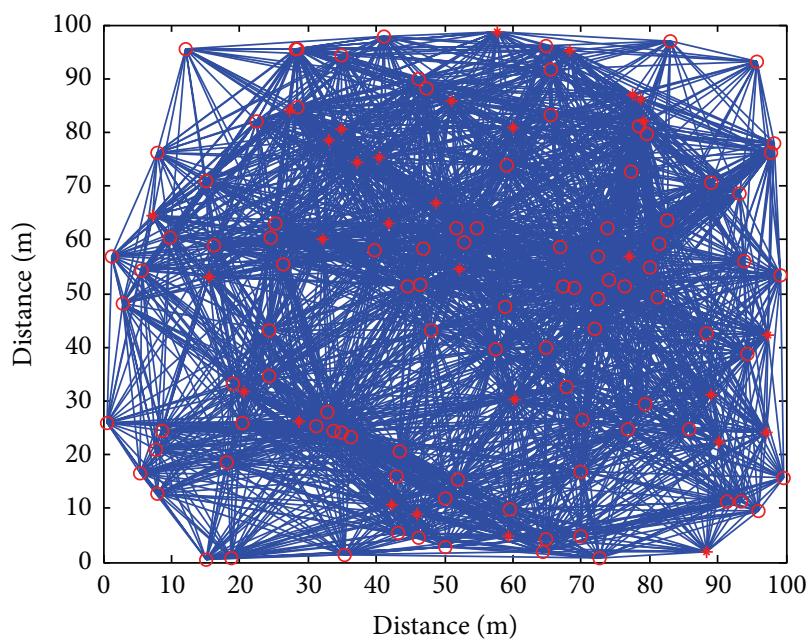

(e)

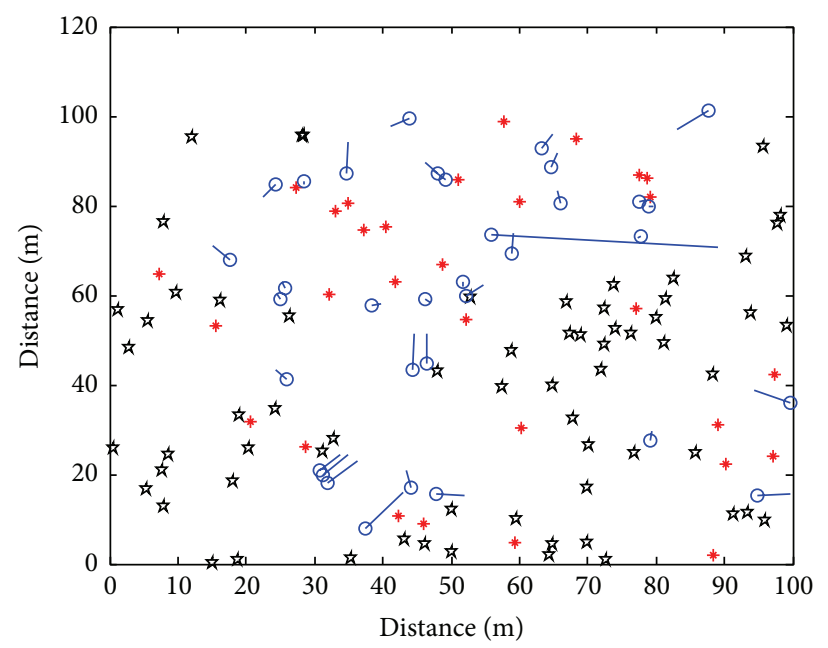

(b)

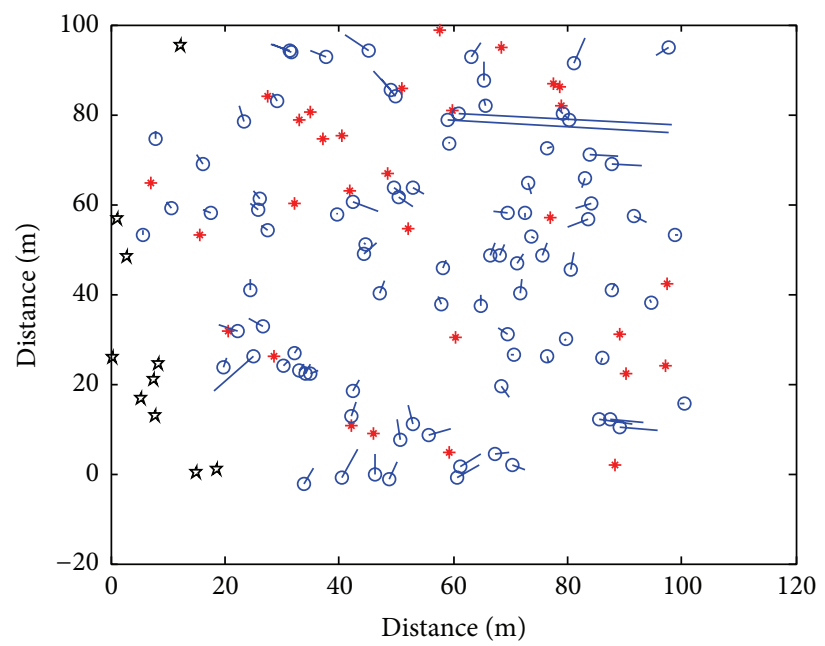

(d)

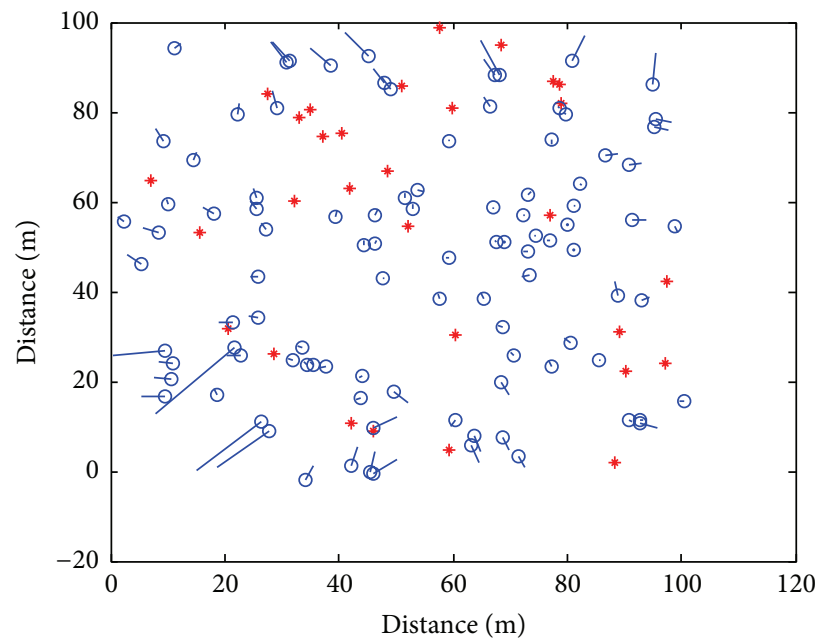

(f)

Figure 6: Connectivity and localization effect ((a), (c), and (e) are the neighbor relationship of nodes for the communication radius of 20 m, $30 \mathrm{~m}$, and $40 \mathrm{~m}$, resp. (b), (d), and (f) are the localization error for the communication radius of $20 \mathrm{~m}, 30 \mathrm{~m}$, and $40 \mathrm{~m}$, resp. The number of beacon nodes is always 30$)$. 
TABLE 2: Contrast of difference mobile paths.

\begin{tabular}{|c|c|c|c|}
\hline Different mobile path planning & Distance of movement & $\begin{array}{c}\text { Nodes of unresolved } \\
\text { localization }\end{array}$ & Localization error \\
\hline $\begin{array}{l}\text { Case 3: path planning algorithm of grid } \\
\text { scan }(\text { communication radius }=30 \mathrm{~m} \text {; } \\
\text { number of beacon nodes }=25)\end{array}$ & 600 & 1 & 0.08767 \\
\hline $\begin{array}{l}\text { Case 4: random way point mobile path } \\
\text { (communication radius }=30 \mathrm{~m} \text {; number } \\
\text { of beacon nodes }=25 \text { ) }\end{array}$ & 329 & 42 & 0.38701 \\
\hline $\begin{array}{l}\text { Case } 5 \text { : random way point mobile path } \\
\text { (communication radius }=30 \mathrm{~m} \text {; number } \\
\text { of beacon nodes }=50 \text { ) }\end{array}$ & 783 & 7 & 0.16785 \\
\hline
\end{tabular}

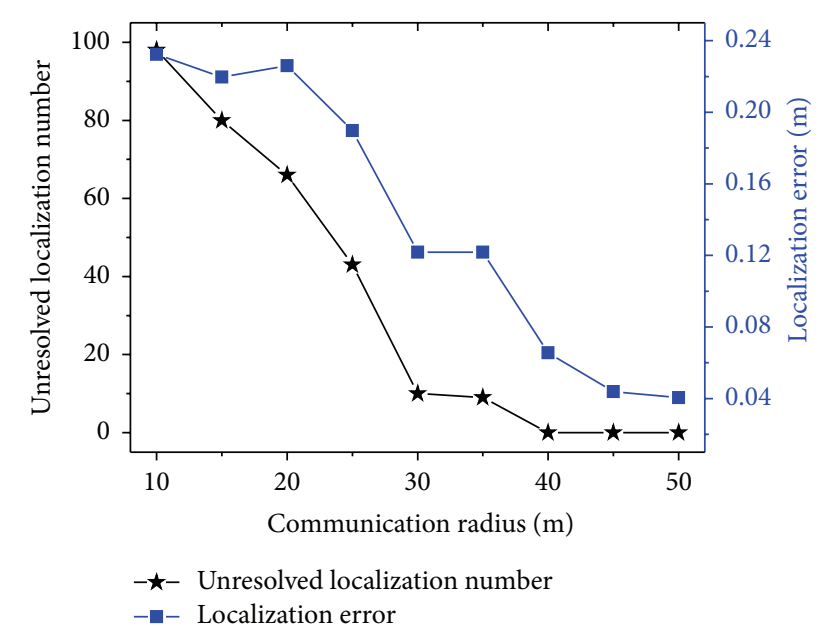

FIGURE 7: Relationship between localization effect and communication radius.

for scheme proposed, whereas there are 28 unresolved localization nodes for (Case 1). Localization error of proposed scheme (Case 2) is obviously lower than localization by beacon deployment randomly. Therefore, it is observed that proposed scheme by a mobile beacon is significantly better than localization scheme by beacon deployment randomly in the connectivity and localization effect in this paper.

Second, to illustrate the localization performance of proposed scheme, we also study the localization scheme by a mobile beacon, compared to the RWP mobile path. For Cases 3 and 4 of Table 2, we set 25 virtual beacon nodes and $30 \mathrm{~m}$ communication radius with the same unknown nodes in the same size sensor field. Scan line divides the square deployment area into $n$ by $n$ subsquares $\left(n=4, l_{g}=5 \mathrm{~m}\right.$ in our case) and connects their vertexes using straight lines as in Figure 9(a). Therefore, the 25 virtual beacon nodes are deployed uniformly in sensor field. To illustrate the advantages of grid scan, we also set 25 nodes (Case 4) and 50 nodes (Case 5) in the RWP mobile path. The beacon mobile path is shown in Figures 9(d) and 9(g), the neighbor relationship of nodes is shown in Figures 9(b), 9(e), and 9(h), and the localization error is shown in Figures 9(c), 9(f), and 9(i).
The results are summarized in Table 2 . The nodes number of unresolved localization is only one for scheme proposed (Case 3), whereas there are 42 and 7 unresolved localization nodes for Cases 4 and 5, respectively. Although RWP scheme can reduce the number of unresolved localization nodes by improving mobile steps, it will cause an increase in the localization time and more network energy cost. The reason is that the virtual beacon nodes are not deployed uniformly among the sensor fields. Localization error of proposed scheme (Case 3) is obviously lower than RWP scheme (Cases 4 and 5).

\section{Conclusions}

In this work, a path planning algorithm based on grid scan which is the entire traverse in sensor field is proposed. In order to improve the localization accuracy, the weighting function is constructed based on the distance between the nodes. Furthermore, an iterative multilateration algorithm is also proposed to avoid decrease in the localization accuracy. At the same time, the start conditions of localization algorithm are also proposed. To evaluate the proposed path planning algorithm, the results of the static beacon randomly deployed and RWP mobile path in sensor field are also provided. It is obtained that proposed scheme by a mobile beacon is significantly better than localization scheme by beacon deployment randomly in localization effects.

\section{Competing Interests}

The authors declare that they have no competing interests.

\section{Acknowledgments}

This research was supported by the National Natural Science Foundation of China (no. 31101080), the China Postdoctoral Science Foundation (no. 2015M580254), the Heilongjiang Postdoctoral Science Foundation (no. LBH-Z15011), Harbin Science and Technology Innovation Youth Talents Special Fund (no. 2015RQQXJ094), "Academic Backbone” Project of Northeast Agricultural University (no. 15XG12), and Northeast Agricultural University Doctoral Start-Up Fund (no. 


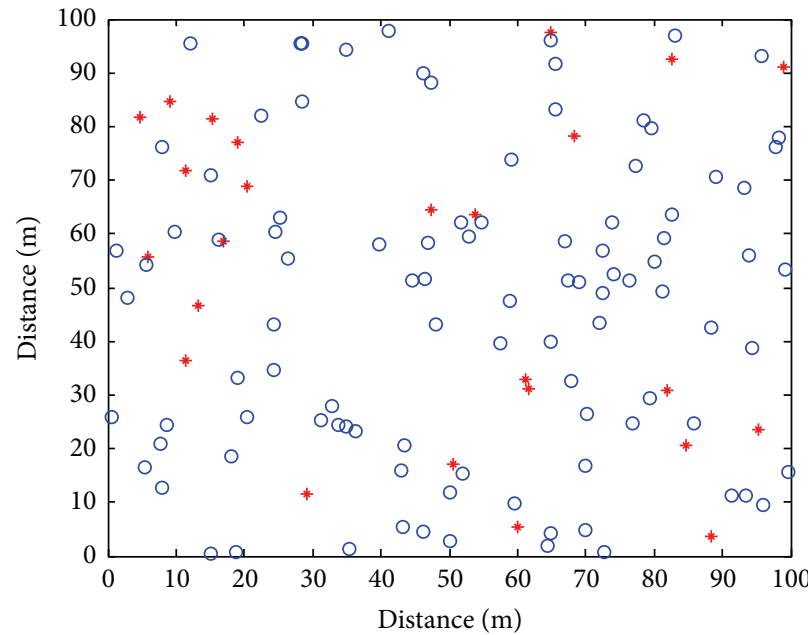

(a)

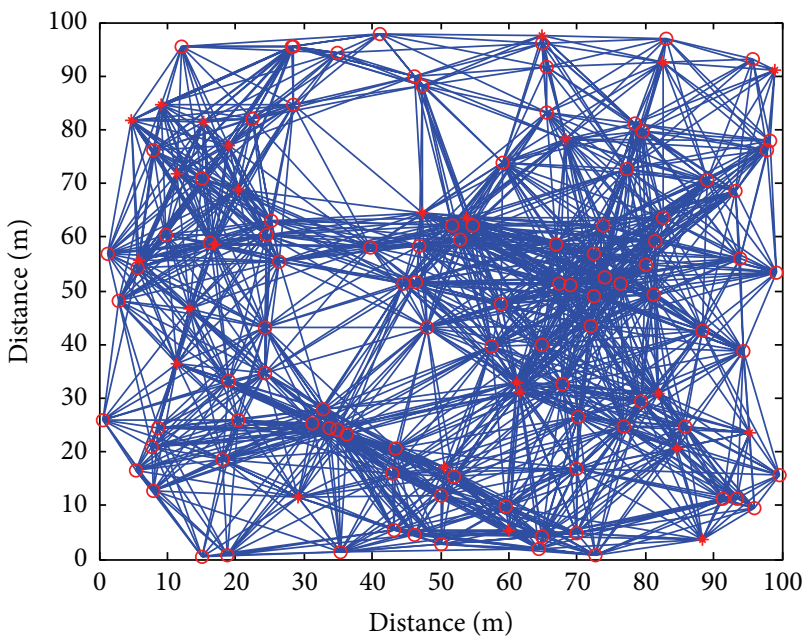

(c)

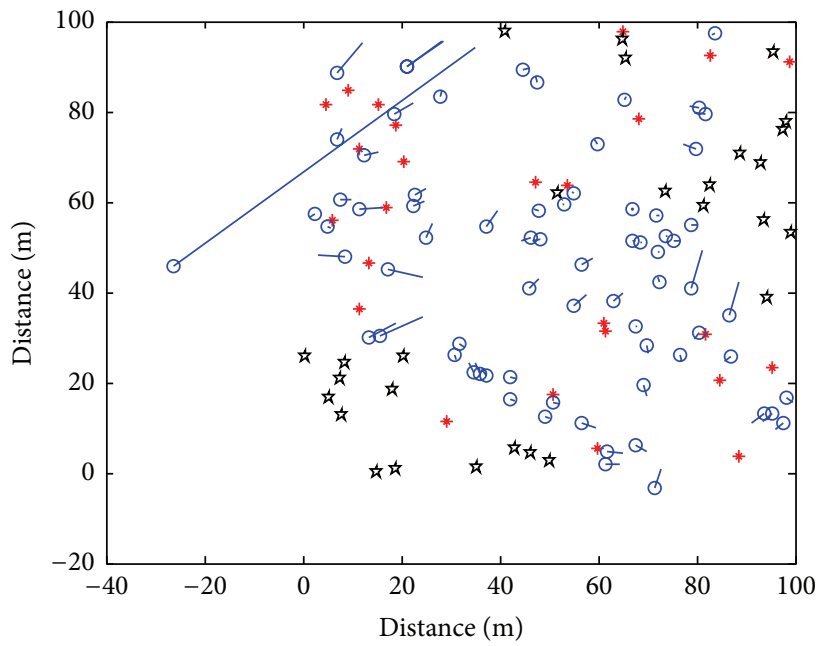

(e)

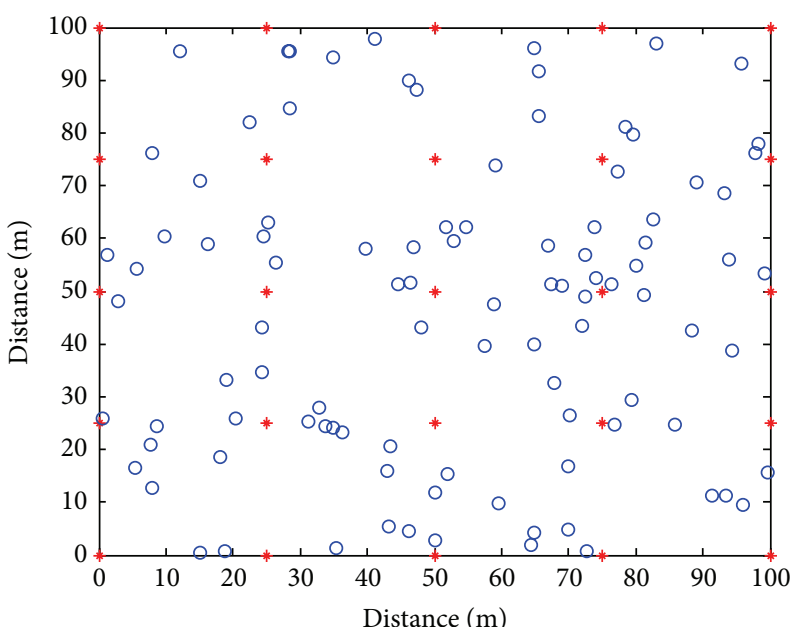

(b)

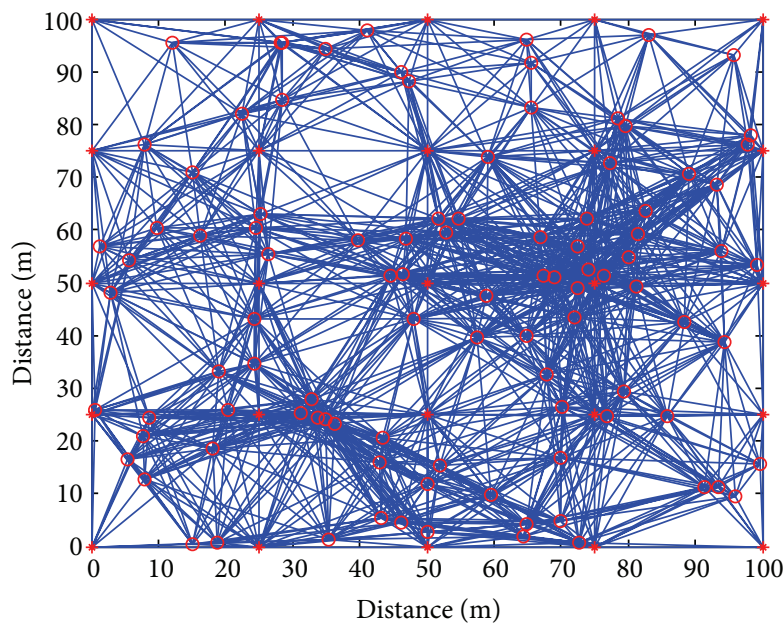

(d)

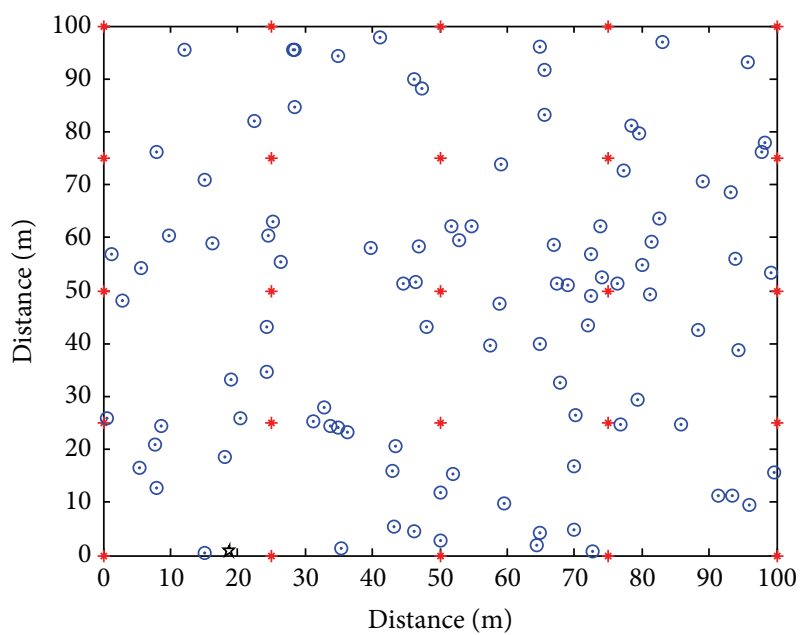

(f)

FIGURE 8: Connectivity and localization effect ((a) and (b) are the node deployment; (c) and (d) are the neighbor relationship of nodes; (e) and (f) are the localization error, in conditions of random deployment and grid scan, resp.). 


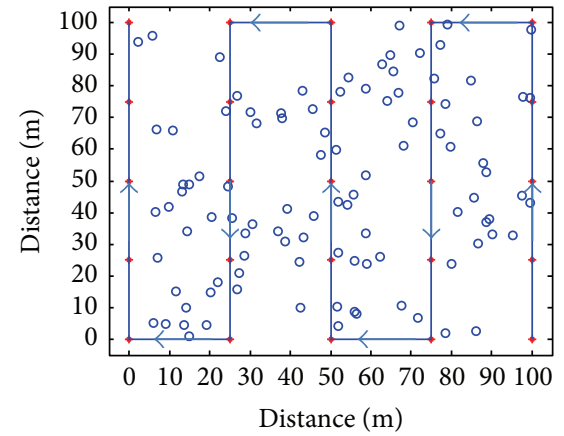

(a)

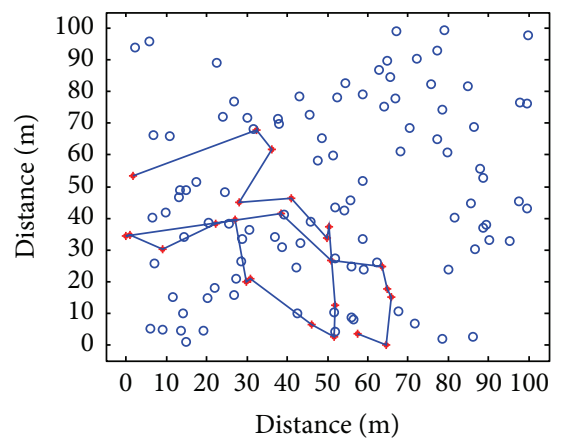

(d)

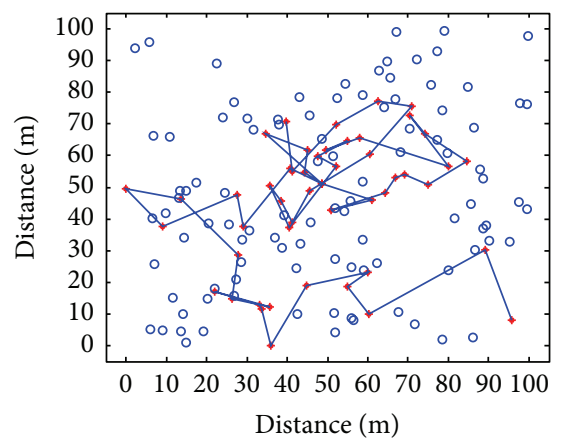

(g)

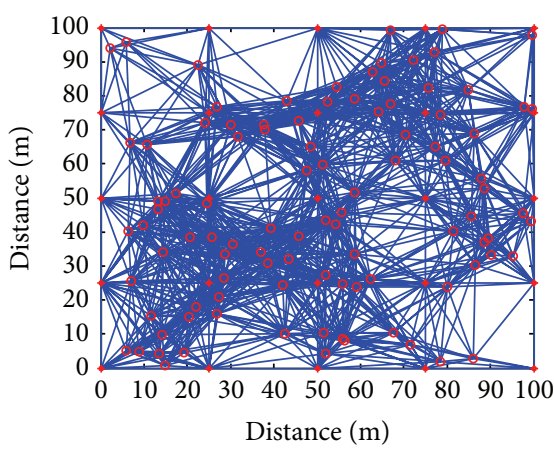

(b)

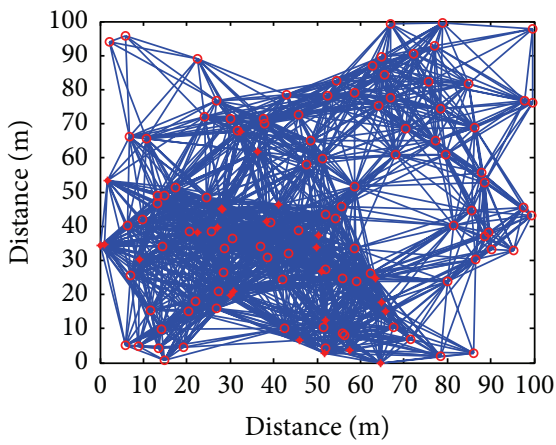

(e)

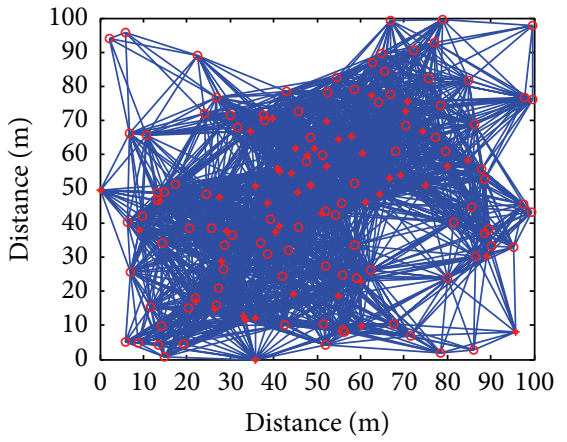

(h)

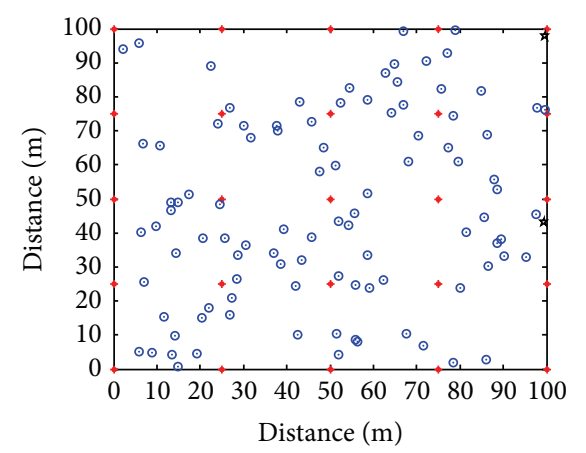

(c)

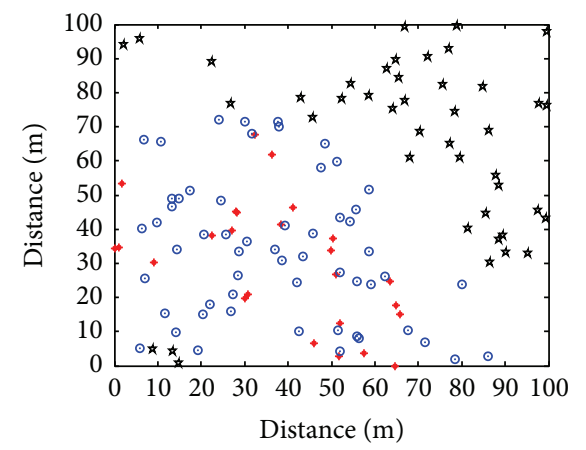

(f)

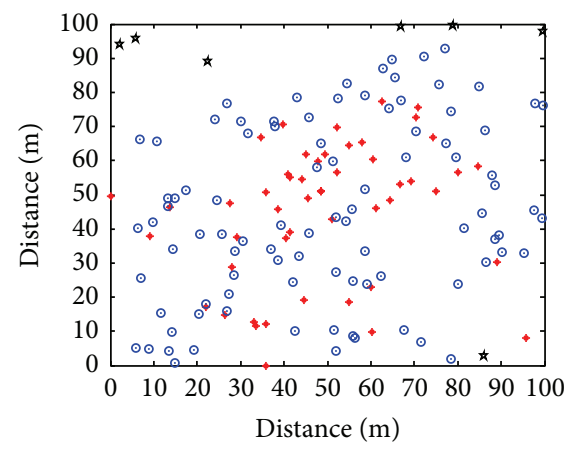

(i)

FIGURE 9: Contrast of difference mobile paths ((a) is the mobile path of grid scan in 25 virtual nodes. (d) and (g) are the mobile path with RWP in 25 and 50 virtual nodes, resp. (b), (e), and (h) are the neighbor relationship of nodes in conditions of grid scan and RWP, resp. (c), (f), and (i) are the localization error in conditions of grid scan and RWP resp. The communication radius is always $30 \mathrm{~m}$ ).

2012RCB51). The authors thank Chengguo Fan, Jiali Du, and Dong Liu of Northeast Agricultural University for their work in revising the paper.

\section{References}

[1] K. Sohraby, D. Minoli, and T. Znati, Wireless Sensor Networks: Technology, Protocols, and Applications, John Wiley \& Sons, London, UK, 2007.

[2] P. Bahl and V. N. Padmanabhan, "Radar: an in-building RFbased user location and tracking system," in Proceedings of IEEE INFOCOM, pp. 775-784, March 2000.

[3] A. Vempaty, O. Ozdemir, K. Agrawal, H. Chen, and P. K. Varshney, "Localization in wireless sensor networks: byzantines and mitigation techniques," IEEE Transactions on Signal Processing, vol. 61, no. 6, pp. 1495-1508, 2013.

[4] N. Bulusu, J. Heidemann, and D. Estrin, "GPS-less low-cost outdoor localization for very small devices," IEEE Personal Communications, vol. 7, no. 5, pp. 28-34, 2000.

[5] J. M. Pak, C. K. Ahn, Y. S. Shmaliy, and M. T. Lim, "Improving reliability of particle filter-based localization in wireless sensor networks via hybrid particle/FIR filtering," IEEE Transactions on Industrial Informatics, vol. 11, no. 5, pp. 1089-1098, 2015.

[6] A. Maddumabandara, H. Leung, and M. Liu, "Experimental evaluation of indoor localization using wireless sensor networks," IEEE Sensors Journal, vol. 15, no. 9, pp. 5228-5237, 2015.

[7] A. Savvides, C. C. Han, and M. B. Strivastava, "Dynamic finegrained localization in ad-hoc networks of sensors," in Proceedings of the 7th Annual International Conference on Mobile 
Computing and Networking, pp. 166-179, ACM, Rome, Italy, July 2001.

[8] Y. Shang and W. Ruml, "Improved MDS-based localization," in Proceedings of the 3rd Annual Joint Conference of the IEEE Computer and Communications Societies (INFOCOM '04), pp. 2640-2651, IEEE, Hong Kong, China, March 2004.

[9] P. N. Pathirana, N. Bulusu, A. V. Savkin, and S. Jha, "Node localization using mobile robots in delay-tolerant sensor networks," IEEE Transactions on Mobile Computing, vol. 4, no. 3, pp. 285296, 2005.

[10] C.-H. Ou, "A localization scheme for wireless sensor networks using mobile anchors with directional antennas," IEEE Sensors Journal, vol. 11, no. 7, pp. 1607-1616, 2011.

[11] B. A. Galstyan, B. Krishnamachari, K. Lerman, and S. Pattem, "Distributed online localization in sensor networks using a moving target," in Proceedings of the 3rd International Symposium on Information Processing in Sensor Networks (IPSN '04), pp. 61-70, Berkeley, Calif, USA, April 2004.

[12] M. L. Sichitiu and V. Ramadurai, "Localization of wireless sensor networks with a mobile beacon," in Proceedings of the Mobile Ad-Hoc and Sensor Systems (MASS '04), pp. 174-183, IEEE, Fort Lauderdale, Fla, USA, October 2004.

[13] J. Rezazadeh, M. Moradi, A. S. Ismail, and E. Dutkiewicz, "Superior path planning mechanism for mobile beacon-assisted localization in wireless sensor networks," IEEE Sensors Journal, vol. 14, no. 9, pp. 3052-3064, 2014.

[14] P. Corke, R. Peterson, and D. Rus, "Networked robots: flying robot navigation using a sensor net," Springer Tracts in Advanced Robotics, vol. 15, pp. 234-243, 2005.

[15] H. Q. Cui and Y. L. Wang, "Four-mobile-beacon assisted localization in three-dimensional wireless sensor networks," Computers and Electrical Engineering, vol. 38, no. 3, pp. 652-661, 2012.

[16] J. Xu and H.-Y. Qian, "Localization of wireless sensor networks with a mobile beacon," Information Technology Journal, vol. 12, no. 11, pp. 2251-2255, 2013.

[17] W. S. Wen and L. Wang, "Path planning of mobile beacon for localization based on distribution of unknown nodes," Advanced Materials Research, vol. 712-715, pp. 1933-1937, 2013.

[18] J.-F. Huang, G.-Y. Chang, and G.-H. Chen, "A historicalbeacon-aided localization algorithm for mobile sensor networks," IEEE Transactions on Mobile Computing, vol. 14, no. 6, pp. 1109-1122, 2015.

[19] Z. Xia and C. Chen, "A localization scheme with mobile beacon for wireless sensor networks," in Proceedings of the 6th International Conference on ITS Telecommunications, pp. 10171020, IEEE, Chengdu, China, June 2006.

[20] G. D. Teng, K. G. Zheng, and G. Yu, "A mobile-beacon-assisted sensor network localization based on RSS and connectivity observations," International Journal of Distributed Sensor Networks, vol. 2011, Article ID 487209, 14 pages, 2011.

[21] K.-F. Ssu, C.-H. Ou, and H. C. Jiau, "Localization with mobile anchor points in wireless sensor networks," IEEE Transactions on Vehicular Technology, vol. 54, no. 3, pp. 1187-1197, 2005.

[22] S. Lee, E. Kim, C. Kim, and K. Kim, "Localization with a mobile beacon based on geometric constraints in wireless sensor networks," IEEE Transactions on Wireless Communications, vol. 8, no. 12, pp. 5801-5805, 2009.

[23] L. Dong and F. L. Severance, "Position estimation with moving beacons in wireless sensor networks," in Proceedings of the IEEE Wireless Communications and Networking Conference (WCNC '07), pp. 2317-2321, IEEE, Hong Kong, March 2007.
[24] K. Kim and W. Lee, "MBAL: a mobile beacon-assisted localization scheme for wireless sensor networks," in Proceedings of the 16th International Conference on Computer Communications and Networks (ICCCN '07), pp. 57-62, IEEE, Honolulu, Hawaii, USA, August 2007.

[25] B. Xiao and H. Chen, "A walking beacon-assisted localization in wireless sensor networks," in Proceedings of the IEEE International Conference on Communications, pp. 3070-3075, Glasgow, UK, June 2007.

[26] G. Teng, K. Zheng, and W. Dong, "MA-MCL: mobile-assisted monte carlo localization for wireless sensor networks," International Journal of Distributed Sensor Networks, vol. 2011, Article ID 671814, 8 pages, 2011.

[27] I. F. Akyildiz and M. C. Vuran, Wireless Sensor Network, John Wiley \& Sons, London, UK, 2010.

[28] N. Patwari, J. N. Ash, S. Kyperountas, A. O. Hero III, R. L. Moses, and N. S. Correal, "Locating the nodes: cooperative localization in wireless sensor networks," IEEE Signal Processing Magazine, vol. 22, no. 4, pp. 54-69, 2005. 


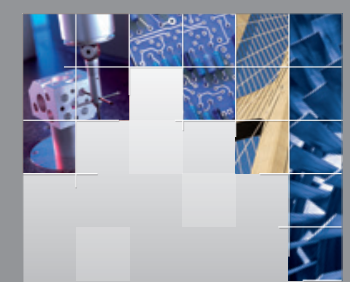

\section{Enfincering}
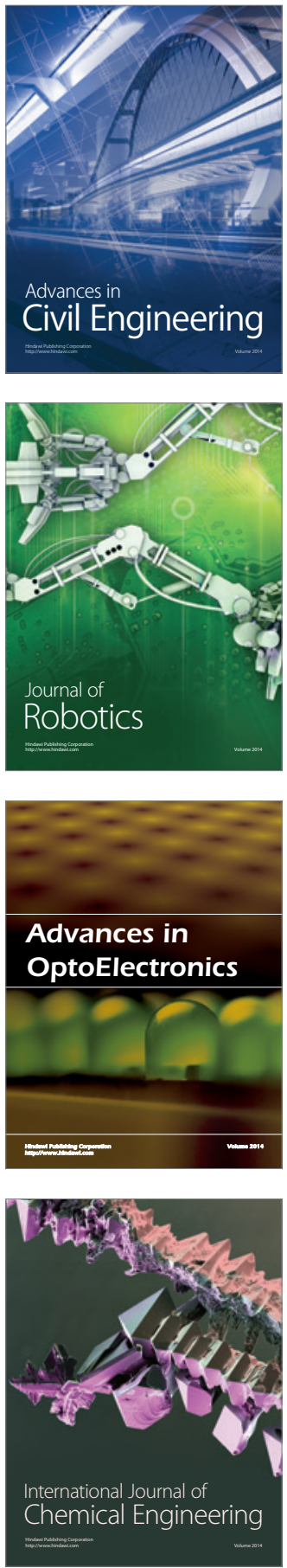

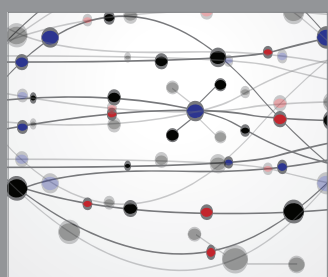

The Scientific World Journal

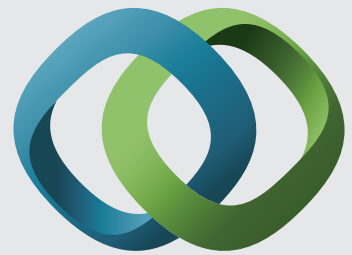

\section{Hindawi}

Submit your manuscripts at

http://www.hindawi.com
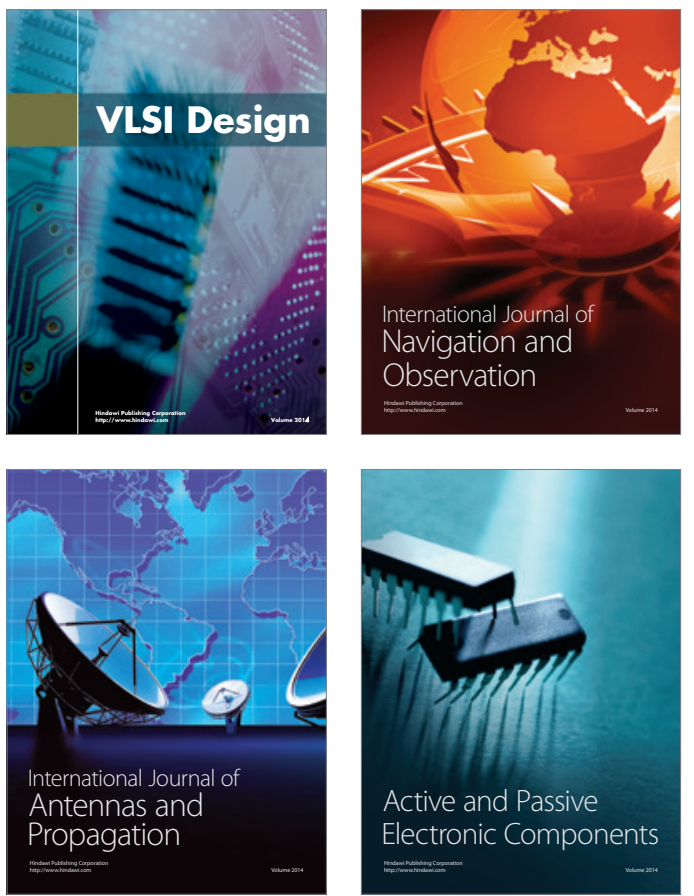
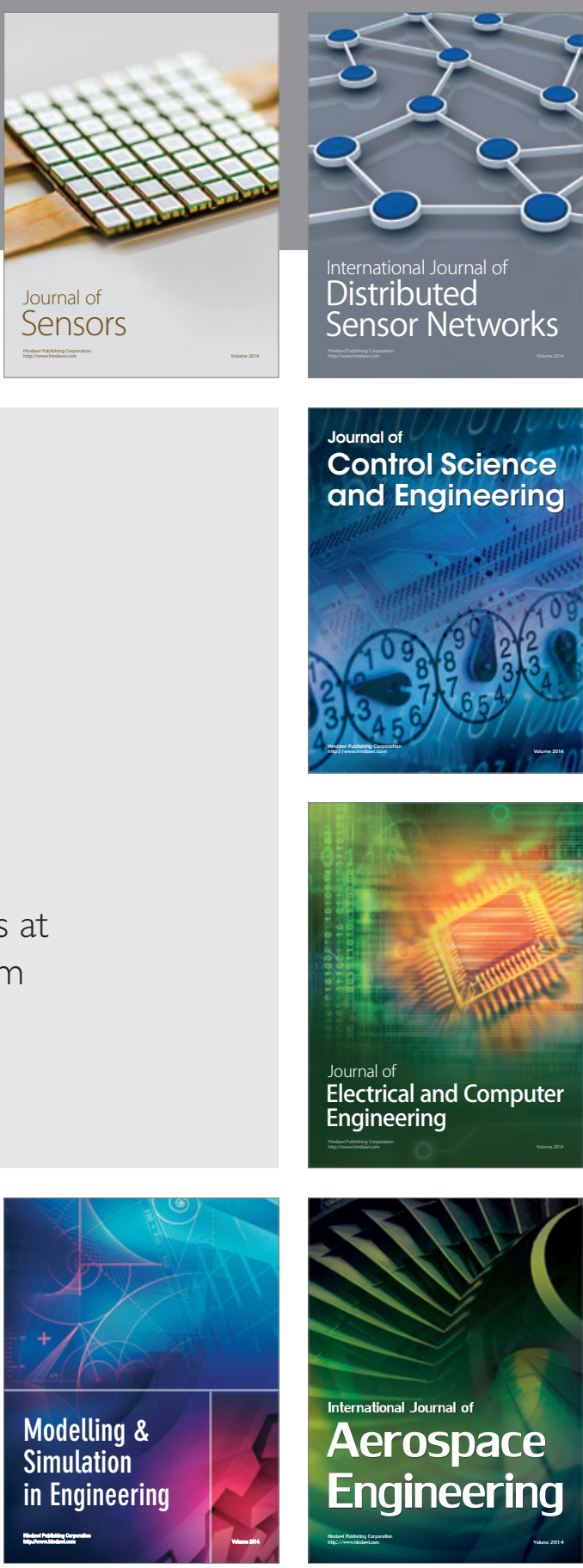

International Journal of

Distributed

Sensor Networks

Journal of

Control Science

and Engineering
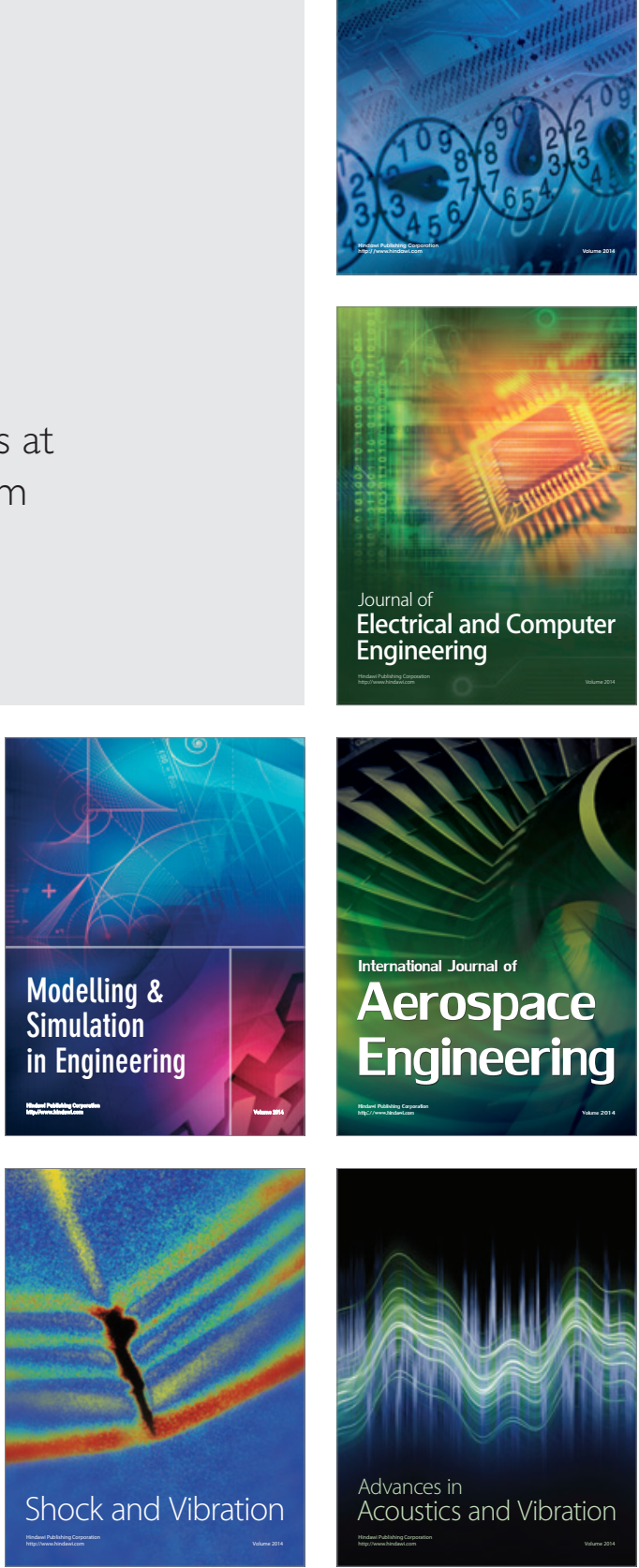